\title{
Elastic least-squares reverse time migration
}

\section{Zongcai Feng*, Gerard T. Schuster, King Abdullah University of Science and Technology}

\section{SUMMARY}

Elastic least-squares reverse time migration (LSRTM) is used to invert synthetic particle-velocity data and crosswell pressure field data. The migration images consist of both the P- and Svelocity perturbation images. Numerical tests on synthetic and field data illustrate the advantages of elastic LSRTM over elastic reverse time migration (RTM). In addition, elastic LSRTM images are better focused and have better reflector continuity than do the acoustic LSRTM images.

\section{INTRODUCTION}

Elastic inversion includes least squares migration and full waveform inversion. It has the potential to construct the P-wave velocity, S-wave velocity, and density models from the recorded data (Tarantola, 1986; Crase et al., 1992). This is not surprising because $\mathrm{P}$ - and S-reflections in the seismic data contain information about the S-velocity and density distributions (Mora, 1987). This suggests that elastic wavefield processing should be used, otherwise the acoustic approximnation can lead to serious artifacts in the migration images (Lu et al., 2009).

Mora (1987) and Tarantola (1986) used the coupled elastic wave equation for prestack depth migration of multi-component seismic data. In addition, Etgen (1988) separated the elastic data into $\mathrm{P}$ and $\mathrm{S}$ potentials and migrated the P-P and P-SV reflections using two migration equations. Later, Yan and Sava (2008) and Du et al. (2012) separated the extrapolated wavefield into $\mathrm{P}$ and $\mathrm{S}$ potentials to provide distinct PP and PS images using crosscorrelation of the vector and scalar potentials.

We choose to invert for the models of the P- and S-wavevelocity perturbations. We use numerical solutions to the elastic wave equation for wavefield extrapolation and use the linearized least-squares inversion method to generate the P- and S-velocity perturbation images.

\section{THEORY}

The 2D velocity-stress elastic wave equation can be written as

$$
\begin{aligned}
& \rho \frac{\partial u_{x}}{\partial t}-\left(\frac{\partial \sigma_{x x}}{\partial x}+\frac{\partial \sigma_{x z}}{\partial z}\right)=0 \\
& \rho \frac{\partial u_{z}}{\partial t}-\left(\frac{\partial \sigma_{z z}}{\partial z}+\frac{\partial \sigma_{x z}}{\partial x}\right)=0 \\
& \frac{\partial \sigma_{x x}}{\partial t}-\lambda\left(\frac{\partial u_{x}}{\partial x}+\frac{\partial u_{z}}{\partial z}\right)-2 \mu \frac{\partial u_{x}}{\partial x}=S_{x x} \\
& \frac{\partial \sigma_{z z}}{\partial t}-\lambda\left(\frac{\partial u_{x}}{\partial x}+\frac{\partial u_{z}}{\partial z}\right)-2 \mu \frac{\partial u_{z}}{\partial z}=S_{z z} \\
& \frac{\partial \sigma_{x z}}{\partial t}-\mu\left(\frac{\partial u_{x}}{\partial z}+\frac{\partial u_{z}}{\partial x}\right)=0
\end{aligned}
$$

Perturbing the background medium parameters $\rho, \lambda$ and $\mu$ by $\delta \rho, \delta \lambda$ and $\delta \mu$, respectively, gives the perturbed wavefield as

$$
\begin{aligned}
& \rho \frac{\partial \delta u_{x}}{\partial t}-\left(\frac{\partial \delta \sigma_{x x}}{\partial x}+\frac{\partial \delta \sigma_{x z}}{\partial z}\right)=0 \\
& \rho \frac{\partial \delta u_{z}}{\partial t}-\left(\frac{\partial \delta \sigma_{z z}}{\partial z}+\frac{\partial \delta \sigma_{x z}}{\partial x}\right)=0, \\
& \frac{\partial \delta \sigma_{x x}}{\partial t}-\lambda\left(\frac{\partial \delta u_{x}}{\partial x}+\frac{\partial \delta u_{z}}{\partial z}\right)-2 \mu \frac{\partial \delta u_{x}}{\partial x} \\
& =\delta \lambda\left(\frac{\partial u_{x}}{\partial x}+\frac{\partial u_{z}}{\partial z}\right)+2 \delta \mu \frac{\partial u_{x}}{\partial x}, \\
& \frac{\partial \delta \sigma_{z z}}{\partial t}-\lambda\left(\frac{\partial \delta u_{x}}{\partial x}+\frac{\partial \delta u_{z}}{\partial z}\right)-2 \mu \frac{\partial \delta u_{z}}{\partial z} \\
& =\delta \lambda\left(\frac{\partial u_{x}}{\partial x}+\frac{\partial u_{z}}{\partial z}\right)+2 \delta \mu \frac{\partial u_{z}}{\partial z}, \\
& \frac{\partial \delta \sigma_{x z}}{\partial t}-\mu\left(\frac{\partial \delta u_{x}}{\partial z}+\frac{\partial \delta u_{z}}{\partial x}\right)=\delta \mu\left(\frac{\partial u_{x}}{\partial z}+\frac{\partial u_{z}}{\partial x}\right) .
\end{aligned}
$$

Equation 2 is equivalent to the matrix-vector operation $\mathbf{d}=\mathbf{L m}$ in least-squares migration (Nemeth et al., 1999). Here, d represents the Born-modeled data where the vertical $u_{x}$ and horizontal $u_{z}$ particle velocities are recorded. $\mathbf{L}$ is a linear modeling operator, and $\mathbf{m}$ is related to the Lamé parameters.

The adjoint equation for equation 1 can be derived using the adjoint-state method (Plessix, 2006):

$\rho \frac{\partial \hat{u}_{x}}{\partial t}-\frac{\partial(\lambda+2 \mu) \hat{\sigma}_{x x}}{\partial x}-\frac{\partial \lambda \hat{\sigma}_{z z}}{\partial x}-\frac{\partial \mu \hat{\sigma}_{x z}}{\partial z}=-\Delta d_{x}\left(\mathbf{x}_{g}, t ; \mathbf{x}_{s}\right)$,

$\rho \frac{\partial \hat{u}_{z}}{\partial t}-\frac{\partial(\lambda+2 \mu) \hat{\sigma}_{z z}}{\partial z}-\frac{\partial \lambda \hat{\sigma}_{x x}}{\partial z}-\frac{\partial \mu \hat{\sigma}_{x z}}{\partial x}=-\Delta d_{z}\left(\mathbf{x}_{g}, t ; \mathbf{x}_{s}\right)$,

$\frac{\partial \hat{\sigma}_{x x}}{\partial t}-\frac{\partial \hat{u}_{x}}{\partial x}=0$,

$\frac{\partial \hat{\sigma}_{z z}}{\partial t}-\frac{\partial \hat{u}_{z}}{\partial z}=0$

$\frac{\partial \hat{\sigma}_{x z}}{\partial t}-\frac{\partial \hat{u}_{x}}{\partial z}-\frac{\partial \hat{u}_{z}}{\partial x}=0$.

Here, $\left(\hat{u}_{x}, \hat{u}_{z}, \hat{\sigma}_{x x}, \hat{\sigma}_{z z}, \hat{\sigma}_{x z}\right)$ are the adjoint-state variables of the state variables $\left(u_{x}, u_{z}, \sigma_{x x}, \sigma_{z z}, \sigma_{x z}\right) . \Delta d_{x}\left(\mathbf{x}_{g}, t ; \mathbf{x}_{s}\right)$ and $\Delta d_{z}\left(\mathbf{x}_{g}, t ; \mathbf{x}_{s}\right)$ represent the residual vertical- and horizontal-particle-velocity seismograms.

The perturbations in the image $\delta \mathbf{m}$ are related to the perturbations of Lamé parameters $\lambda$ and $\mu$. These parameters in turn can be obtained by zeros-lag crosscorrelation of the adjoint fields from equation 3 with the background wavefields from equation 1:

$$
\begin{aligned}
\delta \mathbf{m} & =\left[\begin{array}{l}
\delta \lambda \\
\delta \mu
\end{array}\right] \\
& =\left[\begin{array}{c}
-\int_{0}^{T}\left(\frac{\partial u_{x}}{\partial x}+\frac{\partial u_{z}}{\partial z}\right)\left(\hat{\sigma}_{x x}+\hat{\sigma}_{z z}\right) d t \\
-\int_{0}^{T} 2\left(\frac{\partial u_{x}}{\partial x} \hat{\sigma}_{x x}+\frac{\partial u_{z}}{\partial z} \hat{\sigma}_{z z}\right)+\left(\frac{\partial u_{x}}{\partial z}+\frac{\partial u_{z}}{\partial x}\right) \hat{\sigma}_{x z} d t
\end{array}\right] .
\end{aligned}
$$




\section{Elastic LSRTM}

Equation 4 is equivalent to $\mathbf{g}=\mathbf{L}^{\mathbf{T}} \Delta \mathbf{d}$ in least-squares migration (Nemeth et al., 1999). The perturbation parameters are then used to calculate the $\mathrm{P}$ - and $\mathrm{S}$-wave velocity perturbations as

$$
\begin{aligned}
& \delta v_{P}=2 v_{p} \rho \delta \lambda \\
& \delta v_{S}=-4 v_{S} \rho \delta \lambda+2 v_{S} \rho \delta \mu .
\end{aligned}
$$

Then the model $\mathbf{m}$, can be determined using a least-squares method by minimizing the misfit function, $\varepsilon$, as (Nemeth et al., 1999; Dutta and Schuster, 2014)

$$
\varepsilon=\frac{1}{2}\left\|\mathbf{L m}-\mathbf{d}^{\mathrm{obs}}\right\|^{2},
$$

where $\mathbf{d}^{\text {obs }}$ is the observed data. An iterative conjugate gradient method is used to find the model that minimizes equation 6

\section{NUMERICAL RESULTS}

The effectiveness of elastic LSRTM is now demonstrated with synthetic data and crosswell field data from McElroy, Texas. The synthetic examples are for two land models: (1) a layered model with different P- and S-velocity anomalies and 2) a modified cross section of the SEG/EAGE salt model.

In the synthetic examples, the observed two-component data are generated by an $O(2,8)$ time-space-domain staggered-grid solution of the elastic wave equation in equation 1 without a free-surface condition. The data are then migrated using elastic RTM and elastic LSRTM for the P- and S-velocity perturbations, where the P- and S-images are shown. Here, elastic RTM refers to the first iteration of elastic LSRTM. Source-side illumination is used as the preconditioning factor during the least-squares iterations for elastic LSRTM. The elastic RTM and acoustic LSRTM are also illumination compensated.

\section{Layered velocity model}

We first demonstrate the advantages of elastic LSRTM using a simple example of a flat-layered model. Figure 1 shows a layered model with a shallow P-velocity anomaly on the left and an S-velocity anomaly on the right. The density is homogeneous. To generate the synthetic data, equation 1 is solved for 92 shots evenly spaced at $50 \mathrm{~m}$ on the surface. Here 230 receivers are evenly distributed at $20 \mathrm{~m}$ on the surface. The source-time history is a P-wave source using a Ricker wavelet with a $7.5 \mathrm{~Hz}$ peak frequency and the total recording time is $5.6 \mathrm{~s}$.

Figure 2 compares the elastic RTM and LSRTM images. In both elastic RTM and LSRTM, the S-images have higher resolution than the P-image because of the shorter wavelength of $S$ waves. The elastic LSRTM images have fewer artifacts, better balanced amplitudes and higher resolution compared with the elastic RTM images. In addition, the artifacts from P- and S-velocity anomalies appear in both the P- and S-images of elastic RTM while they are hardly seen in the elastic LSRTM images.

\section{SEG/EAGE salt model}

Elastic LSRTM is now tested on the more complex SEG/EAGE
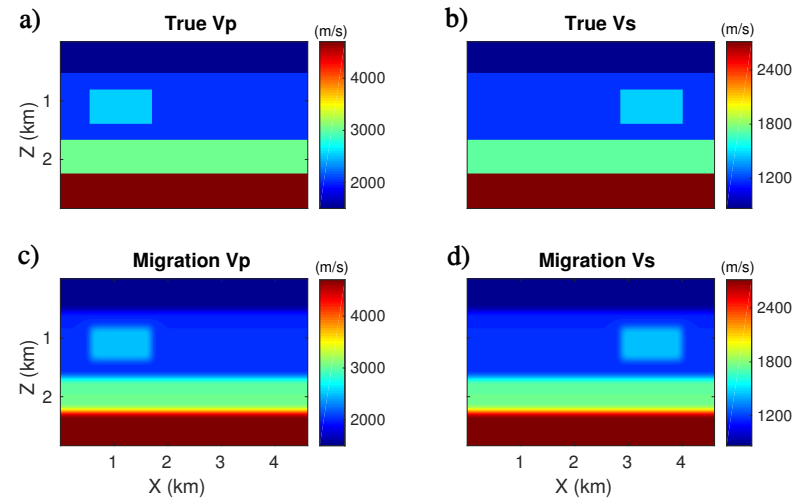

Figure 1: A layered model: (a) true $V_{p}$, (b) true $V_{s}$, (c) migration $V_{p}$, and (d) migration $V_{s}$ models.

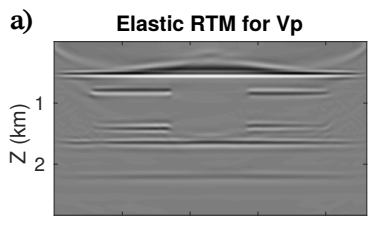

b) Elastic RTM for Vs
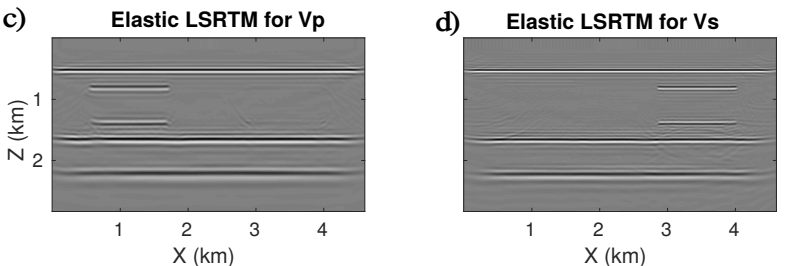

Figure 2: Migration images from elastic RTM (a) for $V_{p}$ and (b) for $V_{s}$, elastic LSRTM (c) for $V_{p}$ and (d) for $V_{s}$.

salt model. Figures $3 \mathrm{a}$ and $3 \mathrm{~b}$ show the true $\mathrm{P}$ - and $\mathrm{S}$-velocity models, respectively. The $\mathrm{S}$-velocity is obtained by scaling the $\mathrm{P}$-velocity by half and the density is homogeneous. The $\mathrm{P}$ - and $\mathrm{S}$-velocity models for migration are shown in Figures $3 \mathrm{c}$ and $3 \mathrm{~d}$, respectively. Equation 1 is solved to generate two particlevelocity components for elastic LSRTM and the pressure component (the negative of the average of the normal stress components) for acoustic LSRTM. 258 shots are evenly spaced at $50 \mathrm{~m}$ and 644 receivers are evenly distributed at $20 \mathrm{~m}$ intervals on the surface. The source-time history is a $\mathrm{P}$-wave source using a Ricker wavelet with a $7.5 \mathrm{~Hz}$ peak frequency and the total recording time is $10 \mathrm{~s}$.

Acoustic and elastic LSRTM images are displayed in Figure 4. The elastic LSRTM images show better resolution and fewer artifacts. In addition, elastic LSRTM shows better images of the salt and subsalt structure compared with the acoustic LSRTM image. The zoom views in Figure 5 show that in elastic LSRTM, the images of the salt interface are more focused and continuous. The zoom views in Figure 6 show that elastic LSRTM improves the subsalt imaging, especially along the steeply dipping events. This is because elastic LSRTM migrates the elastic data into the proper position, especially the converted waves, 


\section{Elastic LSRTM}

which are characterized by a smaller reflection angle compared to the P-P reflections ( $\mathrm{Lu}$ et al., 2009).
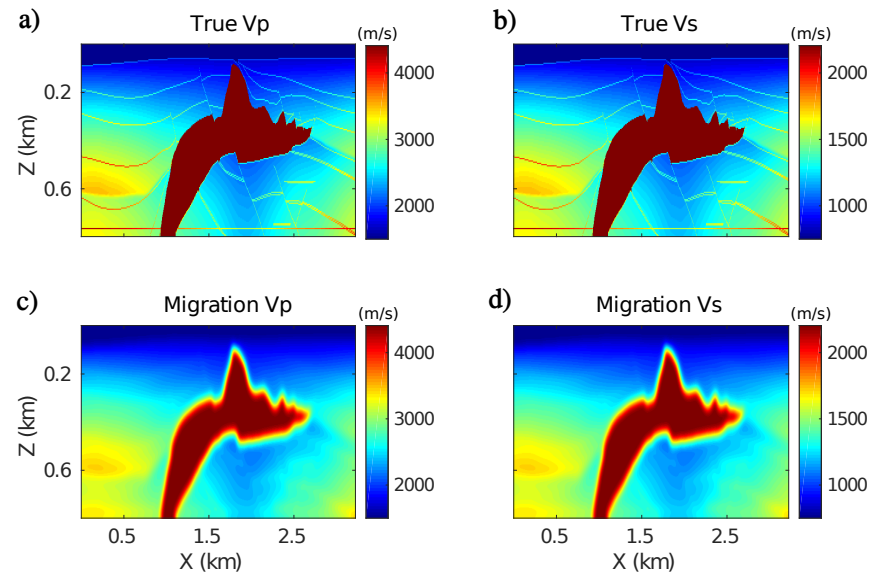

Figure 3: SEG/EAGE salt model: (a) true $V_{p}$, (b) true $V_{s}$, (c) migration $V_{p}$, and (d) migration $V_{s}$ models.
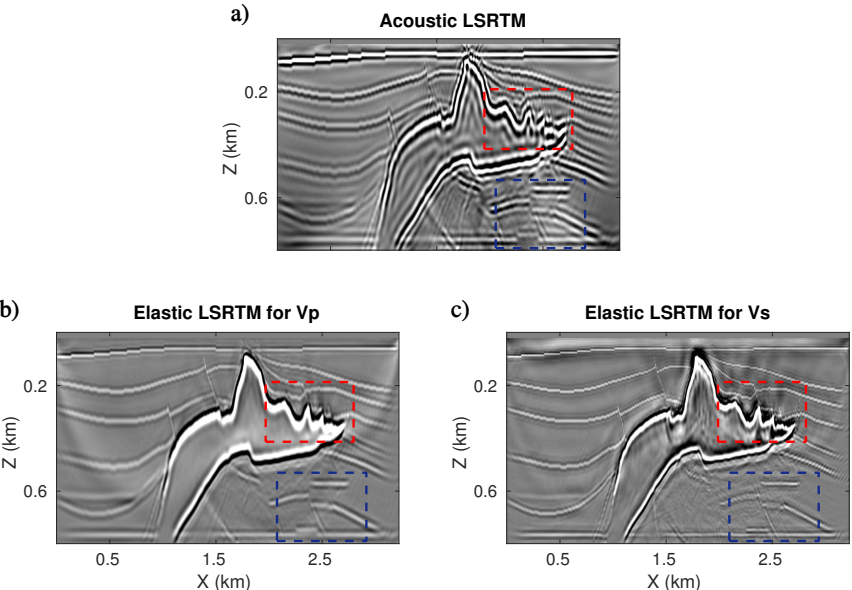

Figure 4: Comparison among images from (a) acoustic LSRTM, elastic LSRTM for (b) $V_{p}$ and (c) for $V_{s}$.

\section{McElroy crosswell field data}

Elastic LSRTM is applied to the McElroy crosswell data (Harris et al., 1992; Zhou et al., 1997). Two 152-m-deep cased wells separated by $56 \mathrm{~m}$ were used as the source and receiver wells. 201 shots were evenly distributed at depth intervals of $0.76 \mathrm{~m}$ from 0 to $152 \mathrm{~m}$ in the source well, and the receiver well had 178 receivers placed at depth intervals of $0.76 \mathrm{~m}$ ranging from 11.4 to $146 \mathrm{~m}$. The data were recorded with a sampling interval of $0.2 \mathrm{~ms}$ for a total recording time of $0.05 \mathrm{~s}$.

A $200-1400 \mathrm{~Hz}$ bandpass filter is applied to the field data to filter out both the high- and low-frequency noise, and a median filter is applied to the common shot gathers to filter out the tube waves generated in the source and receiver wells. Figure 7 shows a common shot gather before and after data processing. The elastic P- and S-wave velocity tomograms (Zhou et al., 1997) shown in Figure 8 are used as the migration velocity
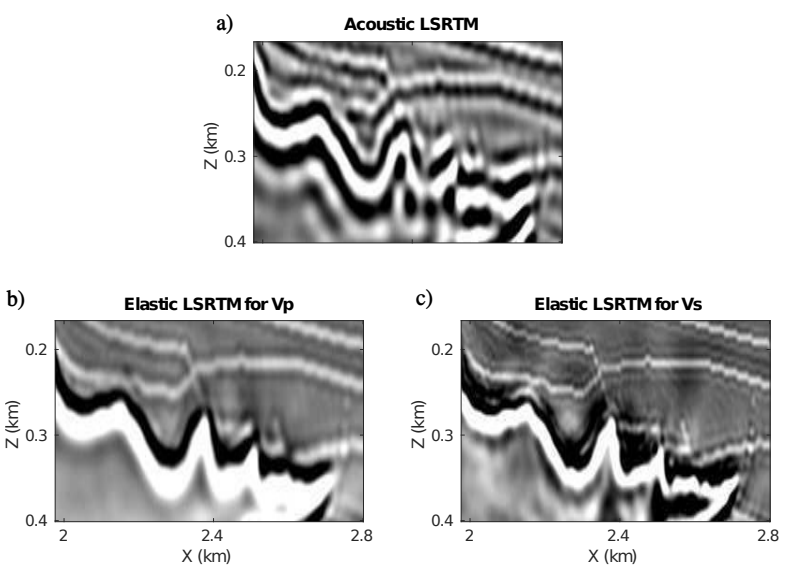

Figure 5: Zoom views of the red boxes showing the salt interface in Figure 4.
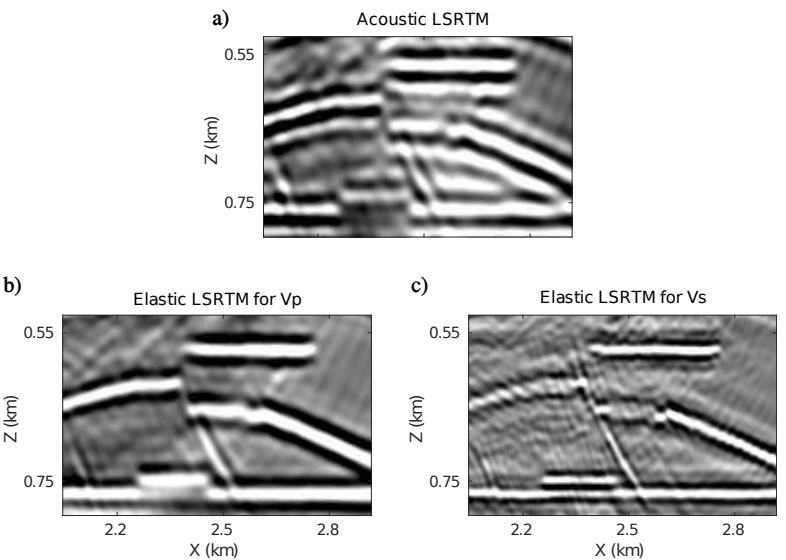

Figure 6: Zoom views of the blue boxes showing the subsalt structure in Figure 4.

model for elastic LSRTM. The direct P-wave are muted out before migration.
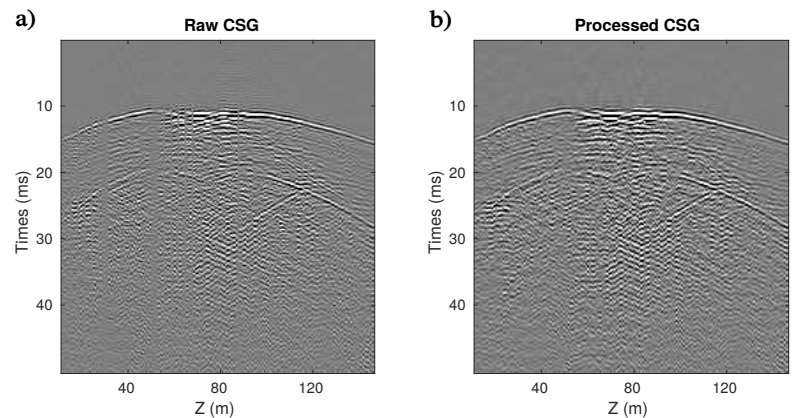

Figure 7: A common shot gather (a) before and (b) after data processing.

We also take the borehole effect (Zhou et al., 1997) into account by the following procedures: 


\section{Elastic LSRTM}
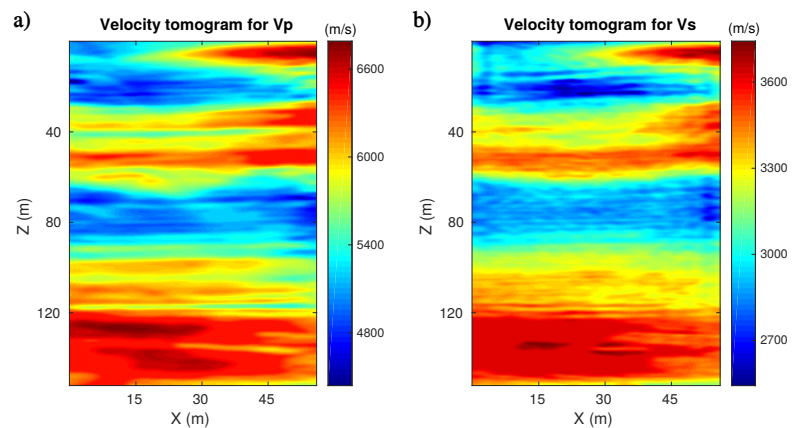

Figure 8: The elastic waveform tomograms for (a) the Pvelocity and (b) S-velocity distributions.

- To account for the strong S-wave events such as S-S, S-P reflections in the data (Harris et al., 1992), the formula for the stress on the wall of the source well is analytically calculated using monopole and dipole seismic sources (White and Lessenger, 1988; Kurkjian et al., 1992; Zhou et al., 1997) to estimate the source radiation pattern:

$$
\begin{aligned}
& \sigma_{x x}(t)=-2 \pi a^{2} \alpha_{T} \frac{\alpha^{2}}{\beta^{2}} \frac{\partial^{2} S(t)}{\partial t^{2}} \\
& \sigma_{z z}(t)=-2 \pi a^{2} \alpha_{T} \frac{\alpha^{2}}{\beta^{2}} \frac{\partial^{2} S(t)}{\partial t^{2}}+4 \pi a^{2} \alpha_{T} \frac{\partial^{2} S(t)}{\partial t^{2}} .
\end{aligned}
$$

Here $\alpha$ and $\beta$ are the $\mathrm{P}$ - and S-wave velocities approximated from the velocity tomograms. The factor $2 \pi a^{2} \alpha_{T}$ is ignored because it just scales the migration images. A Ricker wavelet with a $1200-\mathrm{Hz}$ peak frequency is used to approximate $\frac{\partial^{2} S(t)}{\partial t^{2}}$ for migration.

- The pressure field in the receiver well is calculated from the stress components approximately on the well wall by (White and Lessenger, 1988; Zhou et al., 1997):

$$
P=C\left(\sigma_{x x}-v \sigma_{z z}\right),
$$

where $C$ is an unknown scaling factor and $v$ is the Poisson's ratio calculated from the velocity tomograms. The unknown scaling factor is ignored. The pressure residual is now calculated by:

$$
\Delta \mathbf{d}=P-P^{\mathrm{obs}},
$$

where $P^{\mathrm{obs}}$ is the pressure recorded by the hydrophones in the receiver well.

- The back propagation of the pressure residual requires taking the adjoint operator of equation 8:

$$
\Delta \mathbf{d}^{\mathrm{adj}}=\left[\begin{array}{c}
\Delta \sigma_{x x} \\
\Delta \sigma_{z z}
\end{array}\right]=\left[\begin{array}{c}
1 \\
-v
\end{array}\right] C\left(P-P^{\mathrm{obs}}\right) .
$$

The equation transforms the pressure residual $\Delta \mathbf{d}$ to stress residual $\Delta \mathbf{d}^{\text {adj }}$ before using the $\mathbf{g}=\mathbf{L}^{\mathbf{T}} \Delta \mathbf{d}^{\text {adj }}$ in elastic LSRTM.

Figure 9 compares the elastic RTM and LSRTM images. The P-image of elastic LSRTM has better balanced amplitudes and higher resolution compared with the P-image of elastic RTM. More structures are easier to identify in the P-image of elastic LSRTM especically in the red and blue boxes in Figure 9. However, the S-image of elastic LSRTM has no improvement except for a slightly better amplitude balance compared with elastic RTM. We suspect that there is too much inaccuracy in the S-velocity tomogram (Zhou et al., 1997).
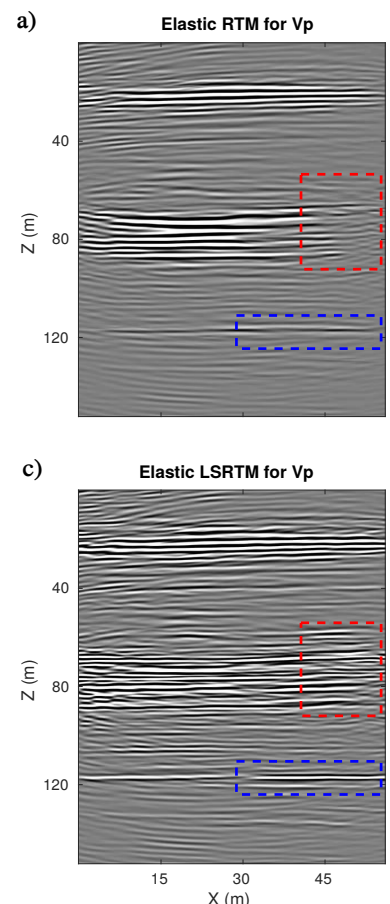

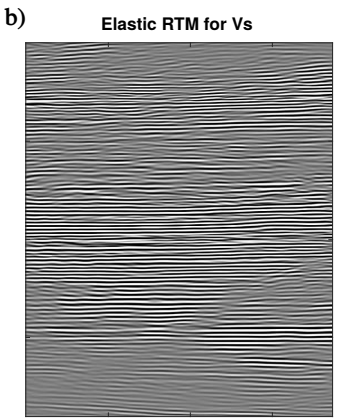

d)

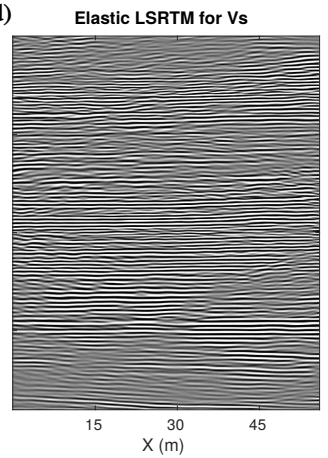

Figure 9: Comparison among images from elastic RTM (a) for $V_{p}$ and (b) for $V_{s}$, elastic LSRTM (c) for $V_{p}$ and (d) for $V_{s}$.

\section{CONCLUSIONS}

Elastic LSRTM is presented for inverting particle velocity data and pressure field data for $\mathrm{P}$ - and S-velocity perturbations. Numerical results show that elastic LSRTM can generate images with fewer artifacts and crosstalk, better balanced amplitudes and higher resolution compared with elastic RTM. Meanwhile, elastic LSRTM improves the the imaging of steeply dipping events and thus generates images with better reflector continuity than does the acoustic LSRTM .

\section{ACKNOWLEDGEMENTS}

We would like to thank the 2015 sponsors of the CSIM Consortium (http://csim.kaust.edu.sa/web/) for their financial support. The computation resource provided by the high performance computing (HPC) center of King Abdullah University of Science and Technology (KAUST) is greatly appreciated. 
EDITED REFERENCES

Note: This reference list is a copyedited version of the reference list submitted by the author. Reference lists for the 2016 SEG Technical Program Expanded Abstracts have been copyedited so that references provided with the online metadata for each paper will achieve a high degree of linking to cited sources that appear on the Web.

\section{REFERENCES}

Crase, E., C. Wideman, M. Noble, and A. Tarantola, 1992, Nonlinear elastic waveform inversion of land seismic reflection data: Journal of Geophysical Research, 97, B4, 4685-4704, http://dx.doi.org/10.1029/90JB00832.

Du, Q., Y. Zhu, and J. Ba, 2012, Polarity reversal correction for elastic reverse time migration: Geophysics, 77, no. 2, S31-S41, http://dx.doi.org/10.1190/geo2011-0348.1.

Dutta, G., and G. T. Schuster, 2014, Attenuation compensation for least-squares reverse time migration using the viscoacoustic-wave equation: Geophysics, 79, no. 6, S251-S262, http://dx.doi.org/10.1190/geo2013-0414.1.

Etgen, J., 1988, Elastic prestack migration of two component data: Geophysics, 289-306.

Harris, J. M., R. NolenHoeksema, I. J. W. Rector, M. V. Schaack, and S. K. Lazaratos, 1992, High resolution crosswell imaging of a west texas carbonate reservoir: Part 1. Data acquisition and project overview, 35-39.

Kurkjian, A. L., H. Schmidt, T. L. Marzetta, J. E. White, and C. Chouzenoux, 1992, Numerical modeling of crosswell seismic monopole sensor data, Annual International Meeting, SEG, Expanded Abstracts, 141-144, http://dx.doi.org/10.1190/1.1822022.

Lu, R., P. Traynin, and J. E. Anderson, 2009, Comparison of elastic and acoustic reversetime migration on the synthetic elastic MarmousiII OBC dataset, 2799-2803.

Mora, P., 1987, Nonlinear two-dimensional elastic inversion of multioffset seismic data: Geophysics, 56, 1211-1228, http://dx.doi.org/10.1190/1.1442384.

Nemeth, T., C. Wu, and G. T. Schuster, 1999, Least-squares migration of incomplete reflection data: Geophysics, 64, 208-221, http://dx.doi.org/10.1190/1.1444517.

Plessix, R.-E., 2006, A review of the adjoint-state method for computing the gradient of a functional with geophysical applications: Geophysical Journal International, 167, 495-503, http://dx.doi.org/10.1111/j.1365-246X.2006.02978.x.

Tarantola, A., 1986, A strategy for nonlinear elastic inversion of seismic reflection data: Geophysics, 51, 1893-1903, http://dx.doi.org/10.1190/1.1442046.

White, J. E., and M. A. Lessenger, 1988, Caliper effect on borehole coupling: Exploration Geophysics, 19, 201-205, http://dx.doi.org/10.1071/EG988201.

Yan, J., and P. Sava, 2008, Isotropic angle-domain elastic reverse-time migration: Geophysics, 73, no. 6, S229-S239, http://dx.doi.org/10.1190/1.2981241.

Zhou, C., G. T. Schuster, S. Hassanzadeh, and J. M. Harris, 1997, Elastic wave equation traveltime and waveform inversion of crosswell data: Geophysics, 62, 853-868, http://dx.doi.org/10.1190/1.1444194. 